\title{
Existence of solutions for the nonlinear multiple base points impulsive fractional differential equations with the three-point boundary conditions
}

Yuxin Hu and Fang Li ${ }^{*}$

*Correspondence:

fangli860@gmail.com

School of Mathematics, Yunnan

Normal University, Kunming,

650092, P.R. China

\begin{abstract}
In this paper, we study a class of nonlinear multiple base points impulsive fractional differential equations involving the three-point boundary conditions. A new result on the existence of a solution is established by using fixed point theorems. An example is presented to illustrate the result.
\end{abstract}

MSC: 34A08; 34A37; 34B10

Keywords: nonlinear multiple base points fractional differential equations; three-point boundary value problem; impulsive condition

\section{Introduction}

The fractional differential equations have received increasing attention during recent years and have been studied extensively (e.g., [1-8] and the references therein). This is mostly due to the fact that fractional calculus provides an efficient and excellent instrument to describe many practical dynamical phenomena which arise in viscoelasticity, electrochemistry, control, porous media, electromagnetic, etc.

In the left and right fractional derivatives ${ }^{c} D_{a^{+}}^{\alpha} x$ and ${ }^{c} D_{b^{-}}^{\alpha} x, a$ and $b$ are called a left base point and a right base point, respectively. Both $a$ and $b$ are called base points of fractional derivatives. A fractional differential equation (FDE) containing more than one base point is called a multiple base points FDE [8]. In this paper, we study the following the threepoint boundary value problem of nonlinear multiple base points fractional differential equations with impulse:

$$
\left\{\begin{array}{l}
{ }^{c} D_{*}^{\alpha} x(t)=f\left(t, x(t),{ }^{c} D_{*}^{\beta} x(t)\right), \quad \text { a.e. } t \in[0, T], \\
\Delta x\left(t_{k}\right)=I_{k}\left(x\left(t_{k}^{-}\right)\right), \quad \Delta x^{\prime}\left(t_{k}\right)=\widetilde{I}_{k}\left(x\left(t_{k}^{-}\right)\right), \quad k=1,2, \ldots, m, \\
x(0)+x(\eta)=0, \quad{ }^{c} D_{0^{+}}^{\gamma_{1}} x(\eta)+{ }^{c} D_{t_{m}^{+}}^{\gamma_{2}} x(T)=0, \quad 0<\eta<t_{1},
\end{array}\right.
$$

where $\alpha \in(1,2), \beta, \gamma_{1}, \gamma_{2} \in(0,1), \alpha-\beta \in(1,2) .{ }^{c} D_{*}^{\alpha}$ is the standard Caputo fractional derivative at the base points $t=t_{k}(k=1,2, \ldots, m)$; that is, $\left.{ }^{c} D_{*}^{\alpha}\right|_{\left.t_{k}, t_{k+1}\right]} x(t)={ }^{c} D_{t_{k}^{+}}^{\alpha} x(t)$ for

(c) The Author(s) 2017. This article is distributed under the terms of the Creative Commons Attribution 4.0 International License (http://creativecommons.org/licenses/by/4.0/), which permits unrestricted use, distribution, and reproduction in any medium, provided you give appropriate credit to the original author(s) and the source, provide a link to the Creative Commons license, and indicate if changes were made. 
all $t \in\left(t_{k}, t_{k+1}\right] . I_{k}, \widetilde{I}_{k} \in C(\mathbb{R}, \mathbb{R}), f: J \times \mathbb{R} \times \mathbb{R} \rightarrow \mathbb{R}$ are appropriate functions to be specified later. The impulsive moments $\left\{t_{k}\right\}$ are given such that $0=t_{0}<t_{1}<\cdots<t_{m}<t_{m+1}=T$, $\Delta x\left(t_{k}\right)$ represents the jump of function $x$ at $t_{k}$, which is defined by $\Delta x\left(t_{k}\right)=x\left(t_{k}^{+}\right)-x\left(t_{k}^{-}\right)$, where $x\left(t_{k}^{+}\right), x\left(t_{k}^{-}\right)$represent the right and left limits of $x(t)$ at $t=t_{k}$ respectively. $\Delta x^{\prime}\left(t_{k}\right)$ has a similar meaning for $x^{\prime}(t)$.

Some recent results on the solvability of boundary value problems of differential equations involving Caputo fractional derivatives can be found in many papers [9-15]. It follows from the definition of fractional order derivative that the solution of (1.1)-(1.3) should belong to the space $A C^{2}\left(\left(t_{k}, t_{k+1}\right], \mathbb{R}\right)(k=0,1, \ldots, m)[1,3]$. However, some researchers neglected this fact $[10,12,13]$. In this paper, we shall provide detailed proofs of our Lemmas 3.2-3.5 which ensure $x(t) \in A C^{2}\left(\left(t_{k}, t_{k+1}\right], \mathbb{R}\right)(k=0,1, \ldots, m)$ to be the solution of $(1.1)$ (1.3) under the weak assumption for $f$. To the best of our knowledge, there has been little study on the existence of solutions for the multiple base points fractional differential equations with impulsive and three-point boundary conditions.

The rest of the paper is organized as follows. In Section 2, we state some basic concepts, notations and preliminary results about fractional calculus. In Section 3, we present the definition of solution for (1.1)-(1.3). In Section 4, by applying some standard fixed point principles, we verify the existence of solutions for problem (1.1)-(1.3). An example is given in Section 5 to demonstrate the application of our main result.

\section{Preliminaries}

In this paper, we denote by $L^{p}(J, \mathbb{R})$ the Banach space of all Lebesgue measurable functions $\xi: J \rightarrow \mathbb{R}$ with the norm $\|\xi\|_{L^{p}}=\left(\int_{J}|\xi(t)|^{p} d t\right)^{\frac{1}{p}}<\infty$ and by $A C([a, b], \mathbb{R})$ the space of all the absolutely continuous functions defined on $[a, b] . A C^{n}([a, b], \mathbb{R})(n=1,2, \ldots)$ is the space of functions $f$ such that $f \in C^{n-1}([a, b], \mathbb{R})$ and $f^{(n-1)} \in A C([a, b], \mathbb{R})$. In particular, $A C^{1}([a, b], \mathbb{R})=A C([a, b], \mathbb{R})$.

Definition 2.1 ([1,3]) The fractional integral of order $\theta$ with the lower limit $a$ for a function $g(t) \in L^{1}([a,+\infty), \mathbb{R})$ is defined as

$$
\left(I_{a^{+}}^{\theta} g\right)(t)=\frac{1}{\Gamma(\theta)} \int_{a}^{t}(t-s)^{\theta-1} g(s) d s, \quad t>a, \theta>0,
$$

where $\Gamma(\cdot)$ is the gamma function.

Definition $2.2([1,3])$ If $g(t) \in A C^{n}([a, b], \mathbb{R})$, then the Riemann-Liouville fractional derivative $\left({ }^{L} D_{a^{+}}^{\theta} g\right)(t)$ of order $\theta$ exists almost everywhere on $[a, b]$ and can be written as

$$
\left({ }^{L} D_{a^{+}}^{\theta} g\right)(t)=\frac{1}{\Gamma(n-\theta)} \frac{d^{n}}{d t^{n}} \int_{a}^{t}(t-s)^{n-\theta-1} g(s) d s, \quad t>a, n-1<\theta<n .
$$

Definition $2.3([1,3])$ If $g(t) \in A C^{n}([a, b], \mathbb{R})$, then the Caputo derivative $\left({ }^{c} D_{a^{+}}^{\theta} g\right)(t)$ of order $\theta$ exists almost everywhere on $[a, b]$ and can be written as

$$
\left({ }^{c} D_{a^{+}}^{\theta} g\right)(t)=\left({ }^{L} D_{a^{+}}^{\theta}\left[g(s)-\sum_{k=0}^{n-1} \frac{g^{(k)}(a)}{k !}(s-a)^{k}\right]\right)(t), \quad t>a, n-1<\theta<n
$$

moreover, if $g(a)=g^{\prime}(a)=\cdots=g^{(n-1)}(a)=0$, then $\left({ }^{c} D_{a^{+}}^{\theta} g\right)(t)=\left({ }^{L} D_{a^{+}}^{\theta} g\right)(t)$. 
$\operatorname{Remark} 2.4([1,3])$ If $g(t) \in C^{n}([a, b], \mathbb{R})$, then $\left({ }^{c} D_{a^{+}}^{\theta} g\right)(t) \in C([a, b], \mathbb{R}), n-1<\theta<n$.

We present here some properties of the fractional calculus as follows.

Lemma $2.5([1,3])$ For $\theta>0$, a general solution of the fractional differential equation ${ }^{c} D_{a^{+}}^{\theta} u(t)=0$ is given by

$$
u(t)=c_{0}+c_{1}(t-a)+c_{2}(t-a)^{2}+\cdots+c_{n-1}(t-a)^{n-1},
$$

where $c_{i} \in \mathbb{R}, i=0,1,2, \ldots, n-1(n=[\theta]+1)$, and $[\theta]$ denotes the integer part of the real number $\theta$.

Lemma $2.6([1,3])$ The Caputo fractional derivative of order $n-1<\theta<n$ for $t^{s}$ is given as

$$
{ }^{c} D_{a^{+}}^{\theta} t^{s}=\left\{\begin{array}{l}
\frac{\Gamma(s+1)}{\Gamma(s-\theta+1)}(t-a)^{s-\theta}, \quad s \in \mathbb{N}, s \geq n \text { or } s \notin \mathbb{N}, s>n-1, \\
0, \quad s \in\{0,1, \ldots, n-1\} .
\end{array}\right.
$$

Lemma 2.7 ([1, 3]) If $\theta_{2}>\theta_{1}>0$ and $f \in L^{p}([a, b], \mathbb{R})(1 \leq p \leq \infty)$, then $\left({ }^{c} D_{a^{+}}^{\theta_{1}} I_{a^{+}}^{\theta_{2}} f\right)(t)=$ $\left(I_{a^{+}}^{\theta_{2}-\theta_{1}} f\right)(t)$.

Let $\mathbf{B}(\cdot, \cdot)$ be the beta function, we need the following result.

Lemma 2.8 For $p>0, q>0,0<a<t, \int_{a}^{t}(t-s)^{p-1}(s-a)^{q-1} d s=(t-a)^{p+q-1} \mathbf{B}(p, q)$.

Proof Let $\tau=s-a$, then $\int_{a}^{t}(t-s)^{p-1}(s-a)^{q-1} d s=\int_{0}^{t-a}(t-a-\tau)^{p-1} \tau^{q-1} d \tau=(t-a)^{p+q-1} \times$ $\mathbf{B}(p, q)$.

Lemma 2.9 For $\varsigma \in(0,1]$ and $0<a \leq b,\left|a^{\varsigma}-b^{\varsigma}\right| \leq(b-a)^{\varsigma}$.

\section{Solutions for the boundary value problem}

Set $J_{k}=\left(t_{k}, t_{k+1}\right], k=1, \ldots, m, J_{0}=\left[0, t_{1}\right]$. We define

$$
X=\left\{x:[0, T] \rightarrow \mathbb{R}: x \in C^{1}\left(J_{k}, \mathbb{R}\right) \text { and } x\left(t_{k}^{+}\right), x^{\prime}\left(t_{k}^{+}\right),{ }^{c} D_{t_{k}^{+}}^{\beta} x\left(t_{k}^{+}\right) \text {exist }, k=0,1, \ldots, m\right\}
$$

with the norm

$$
\|x\|_{1}:=\max \left\{\sup _{k=0,1, \ldots, m} \sup _{t \in J_{k}}|x(t)|, \sup _{k=0,1, \ldots, m} \sup _{t \in J_{k}}\left|x^{\prime}(t)\right|, \sup _{k=0,1, \ldots, m} \sup _{t \in J_{k}}\left|{ }^{c} D_{t_{k}}^{\beta} x(t)\right|\right\} .
$$

Obviously, $X$ is a real Banach space.

In this paper, we consider the following assumption.

(H1) $f: J \times \mathbb{R} \times \mathbb{R} \rightarrow \mathbb{R}$ satisfies $f(\cdot, v, w): J \rightarrow \mathbb{R}$ is measurable for all $v, w \in \mathbb{R}$ and $f(t, \cdot, \cdot): \mathbb{R} \times \mathbb{R} \rightarrow \mathbb{R}$ is continuous for a.e. $t \in J$, and there exists a function $\mu \in L^{\frac{1}{\sigma}}\left(J, \mathbb{R}^{+}\right)\left(\sigma \in\left(0, \frac{\alpha-1}{2}\right)\right)$ such that

$$
|f(t, v, w)| \leq \mu(t)\left(|v|^{\lambda_{1}}+|w|^{\lambda_{2}}\right),
$$

where $0<\lambda_{1}<\lambda_{2}<1$ are real numbers. 
Definition 3.1 A function $x:[0, T] \rightarrow \mathbb{R}$ is said to be a solution of (1.1)-(1.3) if

(1) $x \in A C^{2}\left(J_{k}, \mathbb{R}\right)$;

(2) $x$ satisfies the equation ${ }^{c} D_{t_{k}^{+}}^{\alpha} x(t)=f\left(t, x(t),{ }^{c} D_{t_{k}^{+}}^{\beta} x(t)\right)$ a.e. on $J_{k}$;

(3) for $k=1,2, \ldots, m, \Delta x\left(t_{k}\right)=I_{k}\left(x\left(t_{k}^{-}\right)\right), \Delta x^{\prime}\left(t_{k}\right)=\widetilde{I}_{k}\left(x\left(t_{k}^{-}\right)\right)$and $x(0)+x(\eta)=0$, ${ }^{c} D_{0^{+}}^{\gamma_{1}} x(\eta)+{ }^{c} D_{t_{m}^{+}}^{\gamma_{2}} x(T)=0$.

Next, we present the following lemmas.

Lemma 3.2 For any $\tau_{2}, \tau_{1} \in J_{k}(k=0,1,2, \ldots, m)$ and $\tau_{2}<\tau_{1}$,

$$
\int_{t_{k}}^{\tau_{2}}\left[\left(\tau_{2}-s\right)^{\alpha-2}-\left(\tau_{1}-s\right)^{\alpha-2}\right] \mu(s) d s \rightarrow 0, \quad \text { as } \tau_{2} \rightarrow \tau_{1} .
$$

Proof It follows from Hölder's inequality that

$$
\begin{aligned}
& \left|\int_{t_{k}}^{\tau_{2}}\left[\left(\tau_{2}-s\right)^{\alpha-2}-\left(\tau_{1}-s\right)^{\alpha-2}\right] \mu(s) d s\right| \\
& \quad \leq\|\mu\|_{L^{\frac{1}{\sigma}}}\left[\int_{t_{k}}^{\tau_{2}}\left|\left(\tau_{2}-s\right)^{\alpha-2}-\left(\tau_{1}-s\right)^{\alpha-2}\right|^{\frac{1}{1-\sigma}} d s\right]^{1-\sigma} \\
& \quad=(2-\alpha)\|\mu\|_{L^{\frac{1}{\sigma}}}\left(\int_{t_{k}}^{\tau_{2}}\left|\int_{\tau_{2}}^{\tau_{1}}(\zeta-s)^{\alpha-3} d \zeta\right|^{\frac{1}{1-\sigma}} d s\right)^{1-\sigma} \\
& \quad \leq \bar{M}\left[\int_{t_{k}}^{\tau_{2}}\left(\left(\tau_{2}-s\right)^{\theta}-\left(\tau_{1}-s\right)^{\theta}\right) d s\right]^{1-\sigma} \\
& \quad=\frac{\bar{M}}{(1+\theta)^{1-\sigma}}\left[\left(\tau_{1}-\tau_{2}\right)^{1+\theta}-\left(\tau_{1}-t_{k}\right)^{1+\theta}+\left(\tau_{2}-t_{k}\right)^{1+\theta}\right]^{1-\sigma} \\
& \rightarrow 0, \quad \text { as } \tau_{2} \rightarrow \tau_{1},
\end{aligned}
$$

where $\bar{M}>0$ is a constant and $\theta=\frac{\alpha-2-\sigma}{1-\sigma} \in(-1,0)$.

For $y>\sigma$ and $t_{i-1} \in[0, T](i=1, \ldots, m+1)$, from Hölder's inequality, we have

$$
\begin{aligned}
\int_{t_{i-1}}^{t_{i}}\left(t_{i}-s\right)^{y-1} \mu(s) d s & \leq\left(\int_{t_{i-1}}^{t_{i}}\left(t_{i}-s\right)^{\frac{y-1}{1-\sigma}} d s\right)^{1-\sigma}\|\mu\|_{L^{\frac{1}{\sigma}}} \\
& =\left(\frac{1-\sigma}{y-\sigma}\right)^{1-\sigma}\left(t_{i}-t_{i-1}\right)^{y-\sigma}\|\mu\|_{L^{\frac{1}{\sigma}}} .
\end{aligned}
$$

Noting that $\alpha>\alpha-1>\sigma, \alpha-\gamma_{1}>\sigma, \alpha-\gamma_{2}>\sigma$, we have

$$
\begin{aligned}
& \int_{t_{i-1}}^{t_{i}}\left(t_{i}-s\right)^{\alpha-1} \mu(s) d s \leq\left(\frac{1-\sigma}{\alpha-\sigma}\right)^{1-\sigma}\left(t_{i}-t_{i-1}\right)^{\alpha-\sigma}\|\mu\|_{L^{\frac{1}{\sigma}}}, \\
& \int_{t_{i-1}}^{t_{i}}\left(t_{i}-s\right)^{\alpha-2} \mu(s) d s \leq\left(\frac{1-\sigma}{\alpha-1-\sigma}\right)^{1-\sigma}\left(t_{i}-t_{i-1}\right)^{\alpha-1-\sigma}\|\mu\|_{L^{\frac{1}{\sigma}}}, \\
& \int_{t_{i-1}}^{t_{i}}\left(t_{i}-s\right)^{\alpha-\gamma_{1}-1} \mu(s) d s \leq\left(\frac{1-\sigma}{\alpha-\gamma_{1}-\sigma}\right)^{1-\sigma}\left(t_{i}-t_{i-1}\right)^{\alpha-\gamma_{1}-\sigma}\|\mu\|_{L^{\frac{1}{\sigma}}}, \\
& \int_{t_{i-1}}^{t_{i}}\left(t_{i}-s\right)^{\alpha-\gamma_{2}-1} \mu(s) d s \leq\left(\frac{1-\sigma}{\alpha-\gamma_{2}-\sigma}\right)^{1-\sigma}\left(t_{i}-t_{i-1}\right)^{\alpha-\gamma_{2}-\sigma}\|\mu\|_{L^{\frac{1}{\sigma}}} .
\end{aligned}
$$


Lemma 3.3 Assume that (H1) holds. Then, for $x \in X, k=0,1, \ldots, m$,

$$
\left[I_{t_{k}^{+}}^{\alpha} f\left(s, x(s),{ }^{c} D_{t_{k}^{+}}^{\beta} x(s)\right)\right](t) \in A C^{2}\left(\left[t_{k}, t_{k+1}\right], \mathbb{R}\right)
$$

Proof For $t \in\left[t_{k}, t_{k+1}\right]$, from (3.1), we have

$$
\begin{aligned}
& \int_{t_{k}}^{t} \frac{(t-s)^{\alpha-1}}{\Gamma(\alpha)}\left|f\left(s, x(s),{ }^{c} D_{t_{k}^{+}}^{\beta} x(s)\right)\right| d s \\
& \quad \leq \int_{t_{k}}^{t} \frac{(t-s)^{\alpha-1} \mu(s)}{\Gamma(\alpha)} d s\left(\|x\|_{1}^{\lambda_{1}}+\|x\|_{1}^{\lambda_{2}}\right) \\
& \quad \leq\left(\frac{1-\sigma}{\alpha-\sigma}\right)^{1-\sigma} \frac{\left(t-t_{k}\right)^{\alpha-\sigma}}{\Gamma(\alpha)}\|\mu\|_{L^{\frac{1}{\sigma}}}\left(\|x\|_{1}^{\lambda_{1}}+\|x\|_{1}^{\lambda_{2}}\right), \\
& \int_{t_{k}}^{t} \frac{(t-s)^{\alpha-2}}{\Gamma(\alpha-1)}\left|f\left(s, x(s),{ }^{c} D_{t_{k}^{+}}^{\beta} x(s)\right)\right| d s \\
& \quad \leq \int_{t_{k}}^{t} \frac{(t-s)^{\alpha-2} \mu(s)}{\Gamma(\alpha-1)} d s\left(\|x\|_{1}^{\lambda_{1}}+\|x\|_{1}^{\lambda_{2}}\right) \\
& \quad \leq\left(\frac{1-\sigma}{\alpha-1-\sigma}\right)^{1-\sigma} \frac{\left(t-t_{k}\right)^{\alpha-\sigma-1}}{\Gamma(\alpha-1)}\|\mu\|_{L^{\frac{1}{\sigma}}}\left(\|x\|_{1}^{\lambda_{1}}+\|x\|_{1}^{\lambda_{2}}\right),
\end{aligned}
$$

which means that $(t-s)^{\alpha-1} f\left(s, x(s),{ }^{c} D_{t_{k}^{+}}^{\beta} x(s)\right)$ and $(t-s)^{\alpha-2} f\left(s, x(s),{ }^{c} D_{t_{k}^{+}}^{\beta} x(s)\right)$ are Lebesgue integrable with respect to $s \in\left[t_{k}, t_{k+1}\right]$ for all $t \in\left[t_{k}, t_{k+1}\right]$ and $x \in X$.

Obviously, it follows from the definition of derivative for the Lebesgue integration that

$$
\frac{d}{d t}\left(\left[I_{t_{k}^{+}}^{\alpha} f\left(s, x(s),{ }^{c} D_{t_{k}^{+}}^{\beta} x(s)\right)\right](t)\right)=\left[I_{t_{k}^{+}}^{\alpha-1} f\left(s, x(s),{ }^{c} D_{t_{k}^{+}}^{\beta} x(s)\right)\right](t)
$$

is continuous with respect to $t \in\left[t_{k}, t_{k+1}\right]$.

Next, we show that $\left[I_{t_{k}^{+}}^{\alpha-1} f\left(s, x(s),{ }^{c} D_{t_{k}^{+}}^{\beta} x(s)\right)\right](t) \in A C\left(\left[t_{k}, t_{k+1}\right], \mathbb{R}\right)$. In fact, for every finite collection $\left\{\left(a_{i}, b_{i}\right)\right\}_{1 \leq i \leq n}$ on $\left[t_{k}, t_{k+1}\right]$ with $\sum_{i=1}^{n}\left(b_{i}-a_{i}\right) \rightarrow 0$, noting (3.1) and Lemma 3.2, we have

$$
\begin{aligned}
& \sum_{i=1}^{n} \mid \int_{t_{k}}^{b_{i}}\left(b_{i}-s\right)^{\alpha-2} f\left(s, x(s),{ }^{c} D_{t_{k}^{+}}^{\beta} x(s)\right) d s \\
& \quad-\int_{t_{k}}^{a_{i}}\left(a_{i}-s\right)^{\alpha-2} f\left(s, x(s),{ }^{c} D_{t_{k}^{+}}^{\beta} x(s)\right) d s \mid \\
& \leq \sum_{i=1}^{n}\left|\int_{a_{i}}^{b_{i}}\left(b_{i}-s\right)^{\alpha-2} f\left(s, x(s),{ }^{c} D_{t_{k}^{+}}^{\beta} x(s)\right) d s\right| \\
& \quad+\sum_{i=1}^{n} \int_{t_{k}}^{a_{i}}\left|\left[\left(b_{i}-s\right)^{\alpha-2}-\left(a_{i}-s\right)^{\alpha-2}\right] f\left(s, x(s),{ }^{c} D_{t_{k}^{+}}^{\beta} x(s)\right)\right| d s \\
& \leq\left[\sum_{i=1}^{n} \int_{a_{i}}^{b_{i}}\left(b_{i}-s\right)^{\alpha-2} \mu(s) d s\right. \\
& \left.\quad+\sum_{i=1}^{n} \int_{t_{k}}^{a_{i}}\left[\left(a_{i}-s\right)^{\alpha-2}-\left(b_{i}-s\right)^{\alpha-2}\right] \mu(s) d s\right]\left(\|x\|_{1}^{\lambda_{1}}+\|x\|_{1}^{\lambda_{2}}\right)
\end{aligned}
$$




$$
\begin{aligned}
\leq & {\left[\left(\frac{1-\sigma}{\alpha-1-\sigma}\right)^{1-\sigma} \sum_{i=1}^{n}\left(b_{i}-a_{i}\right)^{\alpha-\sigma-1}\|\mu\|_{L^{\frac{1}{\sigma}}}\right.} \\
& \left.+\sum_{i=1}^{n} \int_{t_{k}}^{a_{i}}\left[\left(a_{i}-s\right)^{\alpha-2}-\left(b_{i}-s\right)^{\alpha-2}\right] \mu(s) d s\right]\left(\|x\|_{1}^{\lambda_{1}}+\|x\|_{1}^{\lambda_{2}}\right)
\end{aligned}
$$

$\longrightarrow 0$.

Hence, $\left[I_{t_{k}^{+}}^{\alpha-1} f\left(s, x(s),{ }^{c} D_{t_{k}^{+}}^{\beta} x(s)\right)\right](t)$ is absolutely continuous on $\left[t_{k}, t_{k+1}\right]$. Now, we can see that $\left[I_{t_{k}^{+}}^{\alpha} f\left(s, x(s),{ }^{c} D_{t_{k}^{+}}^{\beta} x(s)\right)\right](t) \in A C^{2}\left(\left[t_{k}, t_{k+1}\right], \mathbb{R}\right)$. Furthermore, for almost all $t \in\left[t_{k}, t_{k+1}\right]$, $\left[{ }^{c} D_{t_{k}^{+}}^{\alpha} I_{t_{k}}^{\alpha} f\left(s, x(s),{ }^{c} D_{t_{k}^{+}}^{\beta} x(s)\right)\right](t)$ exists.

Lemma 3.4 Assume that (H1) holds. Then, for $x \in X, k=0,1, \ldots, m$,

$$
\left[{ }^{c} D_{t_{k}}^{\alpha}+{ }_{t_{k}^{+}}^{\alpha} f\left(s, x(s),{ }^{c} D_{t_{k}^{+}}^{\beta} x(s)\right)\right](t)=f\left(t, x(t),{ }^{c} D_{t_{k}^{+}}^{\beta} x(t)\right), \quad \text { a.e. } t \in J_{k} .
$$

Proof Let $F(\tau, s)=(t-\tau)^{1-\alpha}|\tau-s|^{\alpha-1} \mu(s)$. Clearly, $F(\tau, s)$ is a nonnegative measurable function on $\left[t_{k}, t\right] \times\left[t_{k}, t\right]$, then $\int_{t_{k}}^{t}\left(\int_{t_{k}}^{t} F(\tau, s) d s\right) d \tau=\int_{t_{k}}^{t}\left(\int_{t_{k}}^{t} F(\tau, s) d \tau\right) d s$. Noting (3.1) and Lemma 2.8, we get

$$
\begin{aligned}
& \int_{t_{k}}^{t}\left(\int_{t_{k}}^{t} F(\tau, s) d s\right) d \tau \\
& \quad=\int_{t_{k}}^{t}(t-\tau)^{1-\alpha}\left[\int_{t_{k}}^{\tau}(\tau-s)^{\alpha-1} \mu(s) d s+\int_{\tau}^{t}(s-\tau)^{\alpha-1} \mu(s) d s\right] d \tau \\
& \quad \leq\left(\frac{1-\sigma}{\alpha-\sigma}\right)^{1-\sigma}\|\mu\|_{L^{\frac{1}{\sigma}}} \int_{t_{k}}^{t}(t-\tau)^{1-\alpha}\left(\tau-t_{k}\right)^{\alpha-\sigma} d \tau+\int_{t_{k}}^{t}\left(\int_{\tau}^{t} \mu(s) d s\right) d \tau \\
& \quad=\left(\frac{1-\sigma}{\alpha-\sigma}\right)^{1-\sigma}\|\mu\|_{L^{\frac{1}{\sigma}}}\left(t-t_{k}\right)^{2-\sigma} \mathbf{B}(2-\alpha, \alpha+1-\sigma)+\int_{t_{k}}^{t}\left(\int_{\tau}^{t} \mu(s) d s\right) d \tau .
\end{aligned}
$$

Therefore, $F_{1}(\tau, s)=(t-\tau)^{1-\alpha}|\tau-s|^{\alpha-1} f\left(s, x(s),{ }^{c} D_{t_{k}^{+}}^{\beta} x(s)\right)$ is an integrable function on $\left[t_{k}, t\right] \times$ $\left[t_{k}, t\right]$, then $\int_{t_{k}}^{t} d \tau \int_{t_{k}}^{\tau} F_{1}(\tau, s) d s=\int_{t_{k}}^{t} d s \int_{s}^{t} F_{1}(\tau, s) d \tau$. According to Lemmas 3.3 and 2.8, we have

$$
\begin{aligned}
& \left({ }^{L} D_{t_{k}^{+}}^{\alpha} I_{t_{k}^{+}}^{\alpha} f\left(s, x(s),{ }^{c} D_{t_{k}^{+}}^{\beta} x(s)\right)\right)(t) \\
& \quad=\frac{1}{\Gamma(2-\alpha) \Gamma(\alpha)} \frac{d^{2}}{d t^{2}} \int_{t_{k}}^{t}(t-\tau)^{1-\alpha}\left[\int_{t_{k}}^{\tau}(\tau-s)^{\alpha-1} f\left(s, x(s),{ }^{c} D_{t_{k}^{+}}^{\beta} x(s)\right) d s\right] d \tau \\
& =\frac{1}{\Gamma(2-\alpha) \Gamma(\alpha)} \frac{d^{2}}{d t^{2}} \int_{t_{k}}^{t} d \tau \int_{t_{k}}^{\tau} F_{1}(\tau, s) d s \\
& =\frac{1}{\Gamma(2-\alpha) \Gamma(\alpha)} \frac{d^{2}}{d t^{2}} \int_{t_{k}}^{t} f\left(s, x(s),{ }^{c} D_{t_{k}^{+}}^{\beta} x(s)\right) d s \int_{s}^{t}(t-\tau)^{1-\alpha}(\tau-s)^{\alpha-1} d \tau \\
& =\frac{d^{2}}{d t^{2}} \int_{t_{k}}^{t}(t-s) f\left(s, x(s),{ }^{c} D_{t_{k}^{+}}^{\beta} x(s)\right) d s \\
& =f\left(t, x(t),{ }^{c} D_{t_{k}^{+}}^{\beta} x(t)\right), \quad \text { a.e. } t \in\left[t_{k}, t_{k+1}\right] .
\end{aligned}
$$


Noting (3.3), we have $\left[I_{t_{k}}^{\alpha} f\left(s, x(s),{ }^{c} D_{t_{k}^{+}}^{\beta} x(s)\right)\right]^{(i)}\left(t_{k}^{+}\right)=0, i=0,1$. Then, from Definition 2.3, with $g(t)$ replaced by $\left[I_{t_{k}}^{\alpha} f\left(s, x(s),{ }^{c} D_{t_{k}^{+}}^{\beta} x(s)\right)\right](t)$ and applying (3.6), we derive

$$
\left[{ }^{c} D_{t_{k}^{+}}^{\alpha} I_{t_{k}}^{\alpha} f\left(s, x(s),{ }^{c} D_{t_{k}^{+}}^{\beta} x(s)\right)\right](t)=\left[{ }^{L} D_{t_{k}^{+}}^{\alpha} I_{t_{k}^{+}}^{\alpha} f\left(s, x(s),{ }^{c} D_{t_{k}^{+}}^{\beta} x(s)\right)\right](t)=f\left(t, x(t),{ }^{c} D_{t_{k}^{+}}^{\beta} x(t)\right) .
$$

This completes the proof.

Set $\widehat{C}=\frac{\Gamma\left(2-\gamma_{1}\right) \Gamma\left(2-\gamma_{2}\right)}{\eta^{1-\gamma_{1}} \Gamma\left(2-\gamma_{2}\right)+\left(T-t_{m}\right)^{1-\gamma_{2}} \Gamma\left(2-\gamma_{1}\right)}$.

From Lemmas 3.3 and 3.4, we have the following result.

Lemma 3.5 Assume that (H1) holds. A function $x \in X$ is a solution of problem (1.1)-(1.3) if and only if $x(t)$ is a solution of the following fractional integral equation:

$$
x(t)= \begin{cases}\left(F_{0}^{\alpha} f\right)(t)+\left(P_{0} f\right)(t)+(Q f)(t), & t \in J_{0}, \\ \left(F_{k}^{\alpha} f\right)(t)+\left(P_{k} f\right)(t)+(Q f)(t), & t \in J_{k}, k=1,2, \ldots, m,\end{cases}
$$

where

$$
\begin{aligned}
\left(F_{k}^{\alpha} f\right)(t)= & \int_{t_{k}}^{t} \frac{(t-s)^{\alpha-1}}{\Gamma(\alpha)} f\left(s, x(s),{ }^{c} D_{t_{k}^{+}}^{\beta} x(s)\right) d s, \quad k=0,1, \ldots, m, \\
\left(P_{k} f\right)(t)= & \sum_{i=1}^{k}\left(\left(F_{i-1}^{\alpha} f\right)\left(t_{i}\right)+I_{i}\left(x\left(t_{i}^{-}\right)\right)\right)+\sum_{i=1}^{k}\left(\left(F_{i-1}^{\alpha-1} f\right)\left(t_{i}\right)+\widetilde{I}_{i}\left(x\left(t_{i}^{-}\right)\right)\right)\left(t-t_{i}\right) \\
& -\frac{1}{2}\left(F_{0}^{\alpha} f\right)(\eta), \quad k=1, \ldots, m, \\
(Q f)(t)= & \widehat{C}\left(t-\frac{\eta}{2}\right)\left[-\left(F_{0}^{\alpha-\gamma_{1}} f\right)(\eta)-\left(F_{m}^{\alpha-\gamma_{2}} f\right)(T)\right. \\
& \left.-\frac{\left(T-t_{m}\right)^{1-\gamma_{2}}}{\Gamma\left(2-\gamma_{2}\right)} \sum_{i=1}^{m}\left(\left(F_{i-1}^{\alpha-1} f\right)\left(t_{i}\right)+\widetilde{I}_{i}\left(x\left(t_{i}^{-}\right)\right)\right)\right] \\
\left(P_{0} f\right)(t)= & -\frac{1}{2}\left(F_{0}^{\alpha} f\right)(\eta) .
\end{aligned}
$$

Proof (Necessity) Let $x \in X$ be the solution of (1.1)-(1.3). From Lemma 3.4, ${ }^{c} D_{t_{k}^{+}}^{\alpha} x(t)=$ $f\left(t, x(t),{ }^{c} D_{t_{k}^{+}}^{\beta} x(t)\right)$ implies ${ }^{c} D_{t_{k}^{+}}^{\alpha}\left(x(t)-\left(F_{k}^{\alpha} f\right)(t)\right)=0, k=0,1, \ldots, m$.

If $t \in J_{0}$, then Lemma 2.5, (3.5), Lemmas 2.6 and 2.7 imply

$$
\begin{aligned}
& x(t)=\left(F_{0}^{\alpha} f\right)(t)+c_{0}+c_{1} t, \quad x^{\prime}(t)=\left(F_{0}^{\alpha-1} f\right)(t)+c_{1}, \\
& { }^{c} D_{0^{+}}^{\gamma_{1}} x(\eta)=\left(F_{0}^{\alpha-\gamma_{1}} f\right)(\eta)+\frac{c_{1} \eta^{1-\gamma_{1}}}{\Gamma\left(2-\gamma_{1}\right)}, \\
& x\left(t_{1}^{-}\right)=\left(F_{0}^{\alpha} f\right)\left(t_{1}\right)+c_{0}+c_{1} t_{1}, \quad x^{\prime}\left(t_{1}^{-}\right)=\left(F_{0}^{\alpha-1} f\right)\left(t_{1}\right)+c_{1},
\end{aligned}
$$

for some $c_{0}, c_{1} \in \mathbb{R}$. Applying the boundary condition $x(0)+x(\eta)=0$, we obtain

$$
c_{0}=-\frac{1}{2}\left(F_{0}^{\alpha} f\right)(\eta)-\frac{c_{1} \eta}{2}
$$


If $t \in J_{1}$, then Lemma 2.5 and (3.5) imply

$$
x(t)=\left(F_{1}^{\alpha} f\right)(t)+c_{2}+c_{3}\left(t-t_{1}\right), \quad x^{\prime}(t)=\left(F_{1}^{\alpha-1} f\right)(t)+c_{3},
$$

for some $c_{2}, c_{3} \in \mathbb{R}$. Thus, we have $x\left(t_{1}^{+}\right)=c_{2}, x^{\prime}\left(t_{1}^{+}\right)=c_{3}$. Applying the impulsive condition (1.2), we derive that

$$
c_{2}=\left(F_{0}^{\alpha} f\right)\left(t_{1}\right)+c_{0}+c_{1} t_{1}+I_{1}\left(x\left(t_{1}^{-}\right)\right), \quad c_{3}=\left(F_{0}^{\alpha-1} f\right)\left(t_{1}\right)+\widetilde{I}_{1}\left(x\left(t_{1}^{-}\right)\right)+c_{1} .
$$

Then, for $t \in J_{1}$,

$$
\begin{aligned}
& x(t)=\left(F_{1}^{\alpha} f\right)(t)+\left(F_{0}^{\alpha} f\right)\left(t_{1}\right)+I_{1}\left(x\left(t_{1}^{-}\right)\right)+\left(\left(F_{0}^{\alpha-1} f\right)\left(t_{1}\right)+\widetilde{I}_{1}\left(x\left(t_{1}^{-}\right)\right)\right)\left(t-t_{1}\right)+c_{0}+c_{1} t, \\
& x^{\prime}(t)=\left(F_{1}^{\alpha-1} f\right)(t)+\left(F_{0}^{\alpha-1} f\right)\left(t_{1}\right)+\widetilde{I}_{1}\left(x\left(t_{1}^{-}\right)\right)+c_{1}, \\
& x\left(t_{2}^{-}\right)=\left(F_{1}^{\alpha} f\right)\left(t_{2}\right)+\left(F_{0}^{\alpha} f\right)\left(t_{1}\right)+I_{1}\left(x\left(t_{1}^{-}\right)\right)+\left(\left(F_{0}^{\alpha-1} f\right)\left(t_{1}\right)+\widetilde{I}_{1}\left(x\left(t_{1}^{-}\right)\right)\right)\left(t_{2}-t_{1}\right)+c_{0}+c_{1} t_{2}, \\
& x^{\prime}\left(t_{2}^{-}\right)=\left(F_{1}^{\alpha-1} f\right)\left(t_{2}\right)+\left(F_{0}^{\alpha-1} f\right)\left(t_{1}\right)+\widetilde{I}_{1}\left(x\left(t_{1}^{-}\right)\right)+c_{1} .
\end{aligned}
$$

If $t \in J_{2}$, then Lemma 2.5 and (3.5) imply

$$
x(t)=\left(F_{2}^{\alpha} f\right)(t)+c_{4}+c_{5}\left(t-t_{2}\right), \quad x^{\prime}(t)=\left(F_{2}^{\alpha-1} f\right)(t)+c_{5},
$$

for some $c_{4}, c_{5} \in \mathbb{R}$. Then $x\left(t_{2}^{+}\right)=c_{4}, x^{\prime}\left(t_{2}^{+}\right)=c_{5}$. Applying the impulsive condition (1.2), we obtain

$$
\begin{aligned}
& c_{4}=\sum_{i=1}^{2}\left(\left(F_{i-1}^{\alpha} f\right)\left(t_{i}\right)+I_{i}\left(x\left(t_{i}^{-}\right)\right)\right)+\left(\left(F_{0}^{\alpha-1} f\right)\left(t_{1}\right)+\widetilde{I}_{1}\left(x\left(t_{1}^{-}\right)\right)\right)\left(t_{2}-t_{1}\right)+c_{0}+c_{1} t_{2}, \\
& c_{5}=\sum_{i=1}^{2}\left(\left(F_{i-1}^{\alpha-1} f\right)\left(t_{i}\right)+\widetilde{I}_{i}\left(x\left(t_{i}^{-}\right)\right)\right)+c_{1} .
\end{aligned}
$$

Then, for $t \in J_{2}$,

$$
\begin{aligned}
x(t)= & \left(F_{2}^{\alpha} f\right)(t)+\sum_{i=1}^{2}\left(\left(F_{i-1}^{\alpha} f\right)\left(t_{i}\right)+I_{i}\left(x\left(t_{i}^{-}\right)\right)\right) \\
& +\sum_{i=1}^{2}\left(\left(F_{i-1}^{\alpha-1} f\right)\left(t_{i}\right)+\widetilde{I}_{i}\left(x\left(t_{i}^{-}\right)\right)\right)\left(t-t_{i}\right)+c_{0}+c_{1} t .
\end{aligned}
$$

By repeating the process, for $t \in J_{k}, k=1,2, \ldots, m$, we have

$$
\begin{aligned}
x(t)= & \left(F_{k}^{\alpha} f\right)(t)+\sum_{i=1}^{k}\left(\left(F_{i-1}^{\alpha} f\right)\left(t_{i}\right)+I_{i}\left(x\left(t_{i}^{-}\right)\right)\right) \\
& +\sum_{i=1}^{k}\left(\left(F_{i-1}^{\alpha-1} f\right)\left(t_{i}\right)+\widetilde{I}_{i}\left(x\left(t_{i}^{-}\right)\right)\right)\left(t-t_{i}\right)+c_{0}+c_{1} t .
\end{aligned}
$$

By (3.11), we obtain

$$
x(t)=\left(F_{k}^{\alpha} f\right)(t)+\left(P_{k} f\right)(t)+c_{1}\left(t-\frac{\eta}{2}\right), \quad t \in J_{k}, k=1,2, \ldots, m .
$$


It follows from (2.1) and Lemma 2.7 that we have

$$
\begin{aligned}
{ }^{c} D_{t_{m}^{+}}^{\gamma_{2}} x(T)= & \left(F_{m}^{\alpha-\gamma_{2}} f\right)(T) \\
& +\sum_{i=1}^{m}\left(\left(F_{i-1}^{\alpha-1} f\right)\left(t_{i}\right)+\widetilde{I}_{i}\left(x\left(t_{i}^{-}\right)\right)\right) \frac{\left(T-t_{m}\right)^{1-\gamma_{2}}}{\Gamma\left(2-\gamma_{2}\right)}+\frac{c_{1}\left(T-t_{m}\right)^{1-\gamma_{2}}}{\Gamma\left(2-\gamma_{2}\right)} .
\end{aligned}
$$

Applying the boundary condition (1.3) to (3.10) and (3.13), we get

$$
\begin{aligned}
c_{1}= & \widehat{C}\left[-\left(F_{0}^{\alpha-\gamma_{1}} f\right)(\eta)-\left(F_{m}^{\alpha-\gamma_{2}} f\right)(T)\right. \\
& \left.-\frac{\left(T-t_{m}\right)^{1-\gamma_{2}}}{\Gamma\left(2-\gamma_{2}\right)} \sum_{i=1}^{m}\left(\left(F_{i-1}^{\alpha-1} f\right)\left(t_{i}\right)+\widetilde{I}_{i}\left(x\left(t_{i}^{-}\right)\right)\right)\right] .
\end{aligned}
$$

Now, it is clear that (3.9)-(3.14) imply (3.7).

(Sufficiency) Let $x(t)$ satisfy (3.7). Noting Lemmas 3.4 and 2.6, $\left({ }^{c} D_{t_{k}^{+}}^{\alpha} t=0\right),\left({ }^{c} D_{t_{k}^{+}}^{\alpha} x\right)(t)$ exists and ${ }^{c} D_{t_{k}^{+}}^{\alpha} x(t)=f\left(t, x(t),{ }^{c} D_{t_{k}^{+}}^{\beta} x(t)\right)$ for a.e. $t \in J_{k}(k=0,1, \ldots, m)$. Moreover,

$$
\begin{aligned}
x\left(t_{k}^{+}\right)-x\left(t_{k}^{-}\right)= & \left(F_{k}^{\alpha} f\right)\left(t_{k}^{+}\right)+\left(P_{k} f\right)\left(t_{k}^{+}\right)+(Q f)\left(t_{k}^{+}\right) \\
& -\left[\left(F_{k-1}^{\alpha} f\right)\left(t_{k}^{-}\right)+\left(P_{k-1} f\right)\left(t_{k}^{-}\right)+(Q f)\left(t_{k}^{-}\right)\right] \\
= & -\int_{t_{k-1}}^{t_{k}} \frac{\left(t_{k}-s\right)^{\alpha-1}}{\Gamma(\alpha)} f\left(s, x(s),{ }^{c} D_{t_{k}^{+}}^{\beta} x(s)\right) d s+\left(F_{k-1}^{\alpha} f\right)\left(t_{k}\right)+I_{k}\left(x\left(t_{k}^{-}\right)\right) \\
= & I_{k}\left(x\left(t_{k}^{-}\right)\right),
\end{aligned}
$$

similarly, $x^{\prime}\left(t_{k}^{+}\right)-x^{\prime}\left(t_{k}^{-}\right)=\widetilde{I}_{k}\left(x\left(t_{k}^{-}\right)\right)$. The boundary conditions (1.3) are clearly satisfied, that is, $x(t)$ satisfies (1.1)-(1.3).

\section{Existence result}

In this section, we deal with the existence of solution for problem (1.1)-(1.3). To this end, we consider the following assumption.

(H2) There exist positive constants $l_{k}, \widetilde{l}_{k}$ and $\lambda_{3} \in[0,1), \lambda_{4} \in[0,1]$ such that

$$
\left|I_{k}(u)\right| \leq l_{k}|u|^{\lambda_{3}}, \quad\left|\widetilde{I}_{k}(u)\right| \leq \widetilde{l}_{k}|u|^{\lambda_{4}}, \quad u \in \mathbb{R}, k=1,2, \ldots, m
$$

For convenience, we introduce the following denotations.

$$
\begin{aligned}
& \delta_{y}=\left(\frac{1-\sigma}{y-\sigma}\right)^{1-\sigma} \cdot \frac{T^{y-\sigma}}{\Gamma(y)}\|\mu\|_{L^{\frac{1}{\sigma}}}, \quad y>\sigma, \\
& \widetilde{C}=m\left(1+\frac{\widehat{C} T^{1-\gamma_{2}}}{\Gamma\left(2-\gamma_{2}\right)}\right) \delta_{\alpha-1}+\widehat{C}\left(\delta_{\alpha-\gamma_{1}}+\delta_{\alpha-\gamma_{2}}\right), \\
& \widetilde{M}=\max \left\{\left(m+\frac{3}{2}\right) \delta_{\alpha}, \delta_{\alpha-1}, \delta_{\alpha-\beta}\right\}+\max \left\{1, T, \frac{T^{1-\beta}}{\Gamma(2-\beta)}\right\} \widetilde{C}, \\
& \widehat{M}=\left(1+\frac{\widehat{C} T^{1-\gamma_{2}}}{\Gamma\left(2-\gamma_{2}\right)}\right) \max \left\{1, T, \frac{T^{1-\beta}}{\Gamma(2-\beta)}\right\} \sum_{i=1}^{m} \widetilde{l}_{i} .
\end{aligned}
$$


Theorem 4.1 Assume that (H1) and (H2) are satisfied, then problem (1.1)-(1.3) has at least one solution $x \in X$ if $\lambda_{4} \in[0,1)$, or $\lambda_{4}=1$ with $\widehat{M}<1$.

Proof Define an operator $\Lambda$ on $X$ as follows:

$$
(\Lambda x)(t)= \begin{cases}\left(F_{0}^{\alpha} f\right)(t)+\left(P_{0} f\right)(t)+(Q f)(t), & t \in J_{0}, \\ \left(F_{k}^{\alpha} f\right)(t)+\left(P_{k} f\right)(t)+(Q f)(t), & t \in J_{k}, k=1,2, \ldots, m,\end{cases}
$$

then

$$
(\Lambda x)^{\prime}(t)=\left\{\begin{array}{l}
\left(F_{0}^{\alpha-1} f\right)(t)+(Q f)^{\prime}(t), \quad t \in J_{0}, \\
\left(F_{k}^{\alpha-1} f\right)(t)+\left(P_{k} f\right)^{\prime}(t)+(Q f)^{\prime}(t), \quad t \in J_{k}, k=1,2, \ldots, m
\end{array}\right.
$$

and

$$
\left({ }^{c} D_{t_{k}^{+}}^{\beta} \Lambda x\right)(t)=\left\{\begin{array}{l}
\left(F_{0}^{\alpha-\beta} f\right)(t)+\left({ }^{c} D_{0^{+}}^{\beta} Q f\right)(t), \quad t \in J_{0}, \\
\left(F_{k}^{\alpha-\beta} f\right)(t)+\left({ }^{c} D_{t_{k}^{+}}^{\beta} P_{k} f\right)(t)+\left({ }^{c} D_{t_{k}^{+}}^{\beta} Q f\right)(t), \quad t \in J_{k}, k=1,2, \ldots, m,
\end{array}\right.
$$

where for $t \in J_{k}, k=1, \ldots, m$,

$$
\begin{aligned}
& \left(P_{k} f\right)^{\prime}(t)=\sum_{i=1}^{k}\left(\left(F_{i-1}^{\alpha-1} f\right)\left(t_{i}\right)+\widetilde{I}_{i}\left(x\left(t_{i}^{-}\right)\right)\right), \\
& (Q f)^{\prime}(t)=\widehat{C}\left[-\left(F_{0}^{\alpha-\gamma_{1}} f\right)(\eta)-\left(F_{m}^{\alpha-\gamma_{2}} f\right)(T)-\frac{\left(T-t_{m}\right)^{1-\gamma_{2}}}{\Gamma\left(2-\gamma_{2}\right)}\left(P_{m} f\right)^{\prime}(t)\right] \\
& \left({ }^{c} D_{t_{k}^{+}}^{\beta} P_{k} f\right)(t)=\frac{\left(t-t_{k}\right)^{1-\beta}}{\Gamma(2-\beta)}\left(P_{k} f\right)^{\prime}(t), \\
& \left({ }^{c} D_{t_{k}^{+}}^{\beta} Q f\right)(t)=\frac{\left(t-t_{k}\right)^{1-\beta}}{\Gamma(2-\beta)}(Q f)^{\prime}(t) .
\end{aligned}
$$

From (H1), $\Lambda: X \rightarrow X$ is clearly well defined. It is obvious that the fixed point of $\Lambda$ is the solution of problem (1.1)-(1.3) by Lemma 3.5. Next, we split the proof into several steps to prove the existence of the fixed point of $\Lambda$.

Step 1 . We prove that the operator $\Lambda$ is continuous.

Let $\left\{x_{n}\right\}$ be a sequence such that $x_{n} \rightarrow x$ in $X$, then there exists $\varepsilon>0$ such that $\left\|x_{n}-x\right\|_{1} \leq \varepsilon$ for $n$ sufficiently large. By (H1), we obtain

$$
\left|f\left(t, x_{n}(t),{ }^{c} D_{t_{k}^{+}}^{\beta} x_{n}(t)\right)-f\left(t, x(t),{ }^{c} D_{t_{k}^{+}}^{\beta} x(t)\right)\right| \leq 2 \mu(t)\left[\left(\varepsilon+\|x\|_{1}\right)^{\lambda_{1}}+\left(\varepsilon+\|x\|_{1}\right)^{\lambda_{2}}\right] .
$$

Moreover, $f$ satisfies (H1), for almost every $t \in J$, we get $f\left(t, x_{n}(t),{ }^{c} D_{t_{k}^{+}}^{\beta} x_{n}(t)\right) \rightarrow f(t, x(t)$, $\left.{ }^{c} D_{t_{k}^{+}}^{\beta} x(t)\right)$ as $n \rightarrow \infty$. It follows from (3.2), the Lebesgue dominated convergence theorem and the continuity of $I_{k}, \widetilde{I}_{k}$ that

$$
\left\|\Lambda x_{n}-\Lambda x\right\|_{1} \rightarrow 0, \quad \text { as } n \rightarrow \infty
$$

Now we can see that $\Lambda$ is continuous. 
Step 2. For $r>0$, we define $B_{r}=\left\{x \in X:\|x\|_{1} \leq r\right\}$. For $x \in B_{r}, k=0,1,2, \ldots, m$, from (3.1) and (3.8), we have

$$
\left|\left(F_{k}^{y} f\right)(t)\right| \leq \delta_{y}\left(r^{\lambda_{1}}+r^{\lambda_{2}}\right),
$$

where $y$ can be replaced by $\alpha, \alpha-1, \alpha-\beta, \alpha-\gamma_{1}$ or $\alpha-\gamma_{2}$.

Furthermore, for $t \in J_{k}, k=0,1, \ldots, m$, by (4.1)-(4.4), we get

$$
\begin{aligned}
& \left|\left(F_{k}^{\alpha} f\right)(t)+\left(P_{k} f\right)(t)+(Q f)(t)\right| \\
& \quad \leq\left[\left(m+\frac{3}{2}\right) \delta_{\alpha}+T \widetilde{C}\right]\left(r^{\lambda_{1}}+r^{\lambda_{2}}\right)+\sum_{i=1}^{m} l_{i} r^{\lambda_{3}}+T\left(1+\frac{\widehat{C} T^{1-\gamma_{2}}}{\Gamma\left(2-\gamma_{2}\right)}\right) \sum_{i=1}^{m} \widetilde{l}_{i} r^{\lambda_{4}}, \\
& \left|\left(F_{k}^{\alpha-1} f\right)(t)+\left(P_{k} f\right)^{\prime}(t)+(Q f)^{\prime}(t)\right| \\
& \quad \leq\left(\delta_{\alpha-1}+\widetilde{C}\right)\left(r^{\lambda_{1}}+r^{\lambda_{2}}\right)+\left(1+\frac{\widehat{C} T^{1-\gamma_{2}}}{\Gamma\left(2-\gamma_{2}\right)}\right) \sum_{i=1}^{m} \widetilde{l}_{i} r^{\lambda_{4}}, \\
& \left|\left(F_{k}^{\alpha-\beta} f\right)(t)+\left({ }^{c} D_{t_{k}^{+}}^{\beta} P_{k} f\right)(t)+\left({ }^{c} D_{t_{k}^{+}}^{\beta} Q f\right)(t)\right| \\
& \quad \leq\left(\delta_{\alpha-\beta}+\frac{T^{1-\beta}}{\Gamma(2-\beta)} \widetilde{C}\right)\left(r^{\lambda_{1}}+r^{\lambda_{2}}\right)+\frac{T^{1-\beta}}{\Gamma(2-\beta)}\left(1+\frac{\widehat{C} T^{1-\gamma_{2}}}{\Gamma\left(2-\gamma_{2}\right)}\right) \sum_{i=1}^{m} \widetilde{l}_{i} r^{\lambda_{4}},
\end{aligned}
$$

which implies

$$
\|\Lambda x\|_{1} \leq \widetilde{M}\left(r^{\lambda_{1}}+r^{\lambda_{2}}\right)+r^{\lambda_{3}} \sum_{i=1}^{m} l_{i}+\widehat{M} r^{\lambda_{4}}
$$

Next, we show that there exists some $r>0$ such that $\Lambda B_{r} \subset B_{r}$. Suppose, on the contrary, that for each $r>0$, there exist $\widetilde{x}^{r}(\cdot) \in B_{r}$ and some $\tilde{t} \in J_{k}$ such that $\Upsilon:=$ $\max \left\{\left|\left(\Lambda \tilde{x}^{r}\right)(\tilde{t})\right|,\left|\left(\Lambda \tilde{x}^{r}\right)^{\prime}(\tilde{t})\right|,{ }^{c} D_{t_{k}^{+}}^{\beta}\left(\Lambda \tilde{x}^{r}\right)(\tilde{t}) \mid\right\}>r$. Then

$$
r<\left\|\Lambda \widetilde{x}^{r}\right\|_{1} \leq \widetilde{M}\left(r^{\lambda_{1}}+r^{\lambda_{2}}\right)+r^{\lambda_{3}} \sum_{i=1}^{m} l_{i}+\widehat{M} r^{\lambda_{4}}
$$

Dividing both sides by $r$ and taking $r \rightarrow \infty$, we get

$$
\begin{cases}1 \leq \lim _{r \rightarrow \infty}\left(\tilde{M}\left(r^{\lambda_{1}-1}+r^{\lambda_{2}-1}\right)+r^{\lambda_{3}-1} \sum_{i=1}^{m} l_{i}+\widehat{M} r^{\lambda_{4}-1}\right)=0 & \text { for } \lambda_{4} \in[0,1), \\ 1 \leq \lim _{r \rightarrow \infty}\left[\tilde{M}\left(r^{\lambda_{1}-1}+r^{\lambda_{2}-1}\right)+r^{\lambda_{3}-1} \sum_{i=1}^{m} l_{i}+\widehat{M}\right]=\widehat{M} & \text { for } \lambda_{4}=1 .\end{cases}
$$

This is a contradiction.

Step 3. We prove that $\Lambda B_{r}$ is equicontinuous on a finite closed sub-interval on $J_{k}$ ( $k=$ $0,1, \ldots, m)$. Let $x \in B_{r}$, for any $\tau_{2}, \tau_{1} \in J_{k}(k=0,1,2, \ldots, m)$ and $\tau_{2}<\tau_{1}$, clearly, we have

$$
\begin{aligned}
& \left|\left(F_{k}^{\alpha} f\right)\left(\tau_{1}\right)-\left(F_{k}^{\alpha} f\right)\left(\tau_{2}\right)\right| \\
& \quad=\left|\int_{t_{k}}^{\tau_{1}} \frac{\left(\tau_{1}-s\right)^{\alpha-1}}{\Gamma(\alpha)} f\left(s, x(s),{ }^{c} D_{t_{k}^{+}}^{\beta} x(s)\right) d s-\int_{t_{k}}^{\tau_{2}} \frac{\left(\tau_{2}-s\right)^{\alpha-1}}{\Gamma(\alpha)} f\left(s, x(s),{ }^{c} D_{t_{k}^{+}}^{\beta} x(s)\right) d s\right|
\end{aligned}
$$




$$
\begin{aligned}
\leq & {\left[\frac{1}{\Gamma(\alpha)} \int_{t_{k}}^{\tau_{2}}\left[\left(\tau_{1}-s\right)^{\alpha-1}-\left(\tau_{2}-s\right)^{\alpha-1}\right] \mu(s) d s\right.} \\
& \left.+\frac{1}{\Gamma(\alpha)} \int_{\tau_{2}}^{\tau_{1}}\left(\tau_{1}-s\right)^{\alpha-1} \mu(s) d s\right]\left(r^{\lambda_{1}}+r^{\lambda_{2}}\right) \\
\rightarrow & 0, \quad \text { as } \tau_{2} \rightarrow \tau_{1} .
\end{aligned}
$$

Therefore,

$$
\begin{aligned}
& \left|(\Lambda x)\left(\tau_{1}\right)-(\Lambda x)\left(\tau_{2}\right)\right| \\
& \quad \leq\left|\left(F_{k}^{\alpha} f\right)\left(\tau_{1}\right)-\left(F_{k}^{\alpha} f\right)\left(\tau_{2}\right)\right|+\left|\left(P_{k} f\right)\left(\tau_{1}\right)-\left(P_{k} f\right)\left(\tau_{2}\right)\right|+\left|(Q f)\left(\tau_{1}\right)-(Q f)\left(\tau_{2}\right)\right| \\
& \quad \leq\left|\left(F_{k}^{\alpha} f\right)\left(\tau_{1}\right)-\left(F_{k}^{\alpha} f\right)\left(\tau_{2}\right)\right|+\left[\widetilde{C}\left(r^{\lambda_{1}}+r^{\lambda_{2}}\right)+\left(1+\frac{\widehat{C} T^{1-\gamma_{2}}}{\Gamma\left(2-\gamma_{2}\right)}\right) \sum_{i=1}^{m} \widetilde{l}_{i} r^{\lambda_{4}}\right]\left(\tau_{1}-\tau_{2}\right) \\
& \quad \rightarrow 0, \quad \text { as } \tau_{2} \rightarrow \tau_{1} .
\end{aligned}
$$

Similarly, by Lemma 3.2 and (3.1),

$$
\begin{aligned}
& \left|(\Lambda x)^{\prime}\left(\tau_{1}\right)-(\Lambda x)^{\prime}\left(\tau_{2}\right)\right| \\
& =\left|\left(F_{k}^{\alpha-1} f\right)\left(\tau_{1}\right)-\left(F_{k}^{\alpha-1} f\right)\left(\tau_{2}\right)\right| \\
& \leq \quad\left[\frac{1}{\Gamma(\alpha)} \int_{t_{k}}^{\tau_{2}}\left[\left(\tau_{1}-s\right)^{\alpha-2}-\left(\tau_{2}-s\right)^{\alpha-2}\right] \mu(s) d s\right. \\
& \left.\quad+\frac{1}{\Gamma(\alpha)} \int_{\tau_{2}}^{\tau_{1}}\left(\tau_{1}-s\right)^{\alpha-2} \mu(s) d s\right]\left(r^{\lambda_{1}}+r^{\lambda_{2}}\right) \\
& \rightarrow 0, \quad \text { as } \tau_{2} \rightarrow \tau_{1},
\end{aligned}
$$

and by Lemma 2.9 ,

$$
\begin{aligned}
& \left|\left({ }^{c} D_{t_{k}^{+}}^{\beta} \Lambda x\right)\left(\tau_{1}\right)-\left({ }^{c} D_{t_{k}^{+}}^{\beta} \Lambda x\right)\left(\tau_{2}\right)\right| \\
& \quad \leq\left|\left(F_{k}^{\alpha-\beta} f\right)\left(\tau_{1}\right)-\left(F_{k}^{\alpha-\beta} f\right)\left(\tau_{2}\right)\right| \\
& \quad+\frac{\left(\tau_{1}-\tau_{2}\right)^{1-\beta}}{\Gamma(2-\beta)}\left[\widetilde{C}\left(r^{\lambda_{1}}+r^{\lambda_{2}}\right)+\left(1+\frac{\widehat{C} T^{1-\gamma_{2}}}{\Gamma\left(2-\gamma_{2}\right)}\right) \sum_{i=1}^{m} \widetilde{l}_{i} r^{\lambda_{4}}\right] \\
& \quad \rightarrow 0, \quad \text { as } \tau_{2} \rightarrow \tau_{1} .
\end{aligned}
$$

Hence the set $\left\{(\Lambda x)(\cdot): x \in B_{r}\right\}$ is equicontinuous. From the above steps, it follows that $\Lambda$ is completely continuous. Now, it follows from Schauder's fixed point theorem that $\Lambda$ has a fixed point $x \in B_{r}$, which corresponds to a solution of problem (1.1)-(1.3).

\section{Application}

In this section, we give an example to illustrate the usefulness of our main result. 
Example 5.1 Consider the following impulsive boundary problem of fractional order:

$$
\left\{\begin{array}{l}
{ }^{c} D_{*}^{\frac{3}{2}} x(t)=x^{\frac{1}{3}}(t)+\frac{1}{\sqrt[12]{t}} \sin \left|{ }^{c} D_{*}^{\frac{1}{5}} x(t)\right|^{\frac{1}{2}}, \quad \text { a.e. } t \in(0,1] \backslash\left\{\frac{1}{3}\right\}, \\
\triangle x\left(\frac{1}{3}\right)=\frac{\left|x\left(\frac{1^{-}}{3}\right)\right|^{\frac{1}{6}}}{10+e^{\left|x\left(\frac{1^{-}}{3}\right)\right|},} \quad \Delta x^{\prime}\left(\frac{1}{3}\right)=\frac{\left|x\left(\frac{1^{-}}{3}\right)\right|^{5}}{7+3\left|x\left(\frac{1}{3}^{-}\right)\right|^{4}}, \\
x(0)+x\left(\frac{1}{4}\right)=0, \quad{ }^{c} D_{0^{+}}^{\frac{1}{3}} x\left(\frac{1}{4}\right)+{ }^{c} D_{\frac{1}{3}}^{\frac{1}{4}} x(1)=0 .
\end{array}\right.
$$

Set

$$
\begin{aligned}
& f\left(t, x(t),{ }^{c} D_{*}^{\frac{1}{5}} x(t)\right)=x^{\frac{1}{3}}(t)+\frac{1}{\sqrt[12]{t}} \sin \left|{ }^{c} D_{*}^{\frac{1}{5}} x(t)\right|^{\frac{1}{2}}, \\
& I_{1}\left(x\left(\frac{1}{3}^{-}\right)\right)=\frac{\left|x\left(\frac{1}{3}^{-}\right)\right|^{\frac{1}{6}}}{10+e^{\left|x\left(\frac{1}{3}^{-}\right)\right|}} \\
& \widetilde{I}_{1}\left(x\left(\frac{1}{3}^{-}\right)\right)=\frac{\left|x\left(\frac{1}{3}^{-}\right)\right|^{5}}{7+3\left|x\left(\frac{1}{3}^{-}\right)\right|^{4}} .
\end{aligned}
$$

Obviously,

$$
\begin{aligned}
& \left|f\left(t, x(t),{ }^{c} D_{*}^{\frac{1}{5}} x(t)\right)\right|<\mu(t)\left[|x(t)|^{\frac{1}{3}}+\left|{ }^{c} D_{*}^{\frac{1}{5}} x(t)\right|^{\frac{1}{2}}\right], \\
& \left|I_{1}(x)\right| \leq \frac{1}{10}|x|^{\frac{1}{6}}, \quad\left|\widetilde{I}_{1}(x)\right| \leq \frac{1}{3}|x|,
\end{aligned}
$$

where $\mu(t)=\frac{1}{\sqrt[12]{t}} \in L^{\frac{1}{\sigma}}([0,1])\left(\sigma=\frac{1}{6}\right)$ and $\|\mu\|_{L^{6}}=2^{\frac{1}{6}}$. Assumptions (H1) and (H2) now hold. Noting that $\alpha=\frac{3}{2}, \beta=\frac{1}{5}, \gamma_{1}=\frac{1}{3}, \gamma_{2}=\frac{1}{4}, \sigma=\frac{1}{6}$ and $l_{1}=\frac{1}{10}, \widetilde{l}_{1}=\frac{1}{3}$, we get

$$
\begin{aligned}
& \widehat{C}=\frac{\Gamma\left(2-\gamma_{1}\right) \Gamma\left(2-\gamma_{2}\right)}{\eta^{1-\gamma_{1}} \Gamma\left(2-\gamma_{2}\right)+\left(T-t_{m}\right)^{1-\gamma_{2}} \Gamma\left(2-\gamma_{1}\right)}=\frac{\Gamma\left(\frac{5}{3}\right) \Gamma\left(\frac{7}{4}\right)}{\left(\frac{1}{4}\right)^{\frac{2}{3}} \Gamma\left(\frac{7}{4}\right)+\left(\frac{2}{3}\right)^{\frac{3}{4}} \Gamma\left(\frac{5}{3}\right)} \approx 0.80, \\
& \widehat{M}=\left(1+\frac{\widehat{C} T^{1-\gamma_{2}}}{\Gamma\left(2-\gamma_{2}\right)}\right) \max \left\{1, T, \frac{T^{1-\beta}}{\Gamma(2-\beta)}\right\} \sum_{i=1}^{m} \widetilde{l}_{i}=\frac{1}{3 \Gamma\left(\frac{9}{5}\right)}\left(1+\frac{\widehat{C}}{\Gamma\left(\frac{7}{4}\right)}\right) \approx 0.67<1 .
\end{aligned}
$$

Therefore, due to the fact that all the assumptions of Theorem 4.1 hold, problem (5.1) has at least one solution.

\section{Competing interests}

The authors declare that they have no competing interests.

\section{Authors' contributions}

All the authors contributed equally and significantly in writing this paper. All authors read and approved the final manuscript.

\section{Acknowledgements}

The authors thank the referee for the improvement of the paper. This work was supported partly by the Natural Science Foundation of China $(11561077,11471227,11201413)$, the Natural Science Foundation of Yunnan Province (2013FB034) and the Reserve Talents of Young and Middle-Aged Academic and Technical Leaders of the Yunnan Province.

Received: 18 October 2016 Accepted: 12 February 2017 Published online: 20 February 2017

\section{References}

1. Kilbas, AA, Srivastava, HM, Trujillo, JJ: Theory and Applications of Fractional Differential Equations. North-Holland Mathematics Studies, vol. 204. Elsevier, Amsterdam (2006) 
2. Hernandez, E, O'Regan, D, Balachandran, K: On recent developments in the theory of abstract differential equations with fractional derivatives. Nonlinear Anal. 73(10), 3462-3471 (2010)

3. Podlubny, I: Fractional Differential Equations. Mathematics in Science and Engineering, vol. 198. Academic Press, San Diego (1999)

4. Mophou, GM, N'Guérékata, GM: On some classes of almost automorphic functions and applications to fractional differential equations. Comput. Math. Appl. 59, 1310-1317 (2010)

5. Zhou, Y: Basic Theory of Fractional Differential Equations. World Scientific, Singapore (2014)

6. Wang, G, Ahmad, B, Zhang, L, Nieto, JJ: Comments on the concept of existence of solution for impulsive fractional differential equations. Commun. Nonlinear Sci. Numer. Simul. 19, 401-403 (2014)

7. Lan, KQ, Lin, W: Positive solutions of systems of Caputo fractional differential equations. Commun. Appl. Anal. 17(1), 61-68 (2013)

8. Liu, YJ, Ahmad, B: A study of impulsive multiterm fractional differential equations with single and multiple base points and applications. Sci. World J. 2014, Article ID 194346 (2014)

9. Agarwal, RP, Benchohra, M, Hamani, S: A survey on existence results for boundary value problems of nonlinear fractional differential equations and inclusions. Acta Appl. Math. 109, 973-1033 (2010)

10. Ashyralyev, A, Sharifov, YA: Existence and uniqueness of solutions for the system of nonlinear fractional differential equations with nonlocal and integral boundary conditions. Abstr. Appl. Anal. 2012, Article ID 594802 (2012)

11. Liang, J, Liu, J, Xiao, T: Nonlocal impulsive problems for nonlinear differential equations in Banach spaces. Math. Comput. Model. 49, 798-804 (2009)

12. Bai, Z, Lu, H: Positive solutions for boundary value problem of nonlinear fractional differential equations. J. Math. Anal. Appl. 311, 459-505 (2005)

13. Kaufmann, ER, Mboumi, E: Positive solutions of a boundary value problem for a nonlinear fractional differential equation. Electron. J. Qual. Theory Differ. Equ. 3, 11 (2008)

14. Ahmad, B, Nieto, JJ: Existence of solutions for impulsive anti-periodic boundary value problems of fractional order. Taiwan. J. Math. 15(3), 981-993 (2011)

15. Wang, G, Ahmad, B, Zhang, L: Impulsive anti-periodic boundary value problem for nonlinear differential equations of fractional order. Nonlinear Anal. TMA 74, 792-804 (2011)

\section{Submit your manuscript to a SpringerOpen ${ }^{\circ}$ journal and benefit from:}

- Convenient online submission

Rigorous peer review

- Immediate publication on acceptance

Open access: articles freely available online

- High visibility within the field

- Retaining the copyright to your article 